\title{
REFLEXIONES SOBRE LA RELACIÓN MÉDICO PACIENTE
}

\author{
REFLECTIONS ON THE PATIENT MEDICAL RELATIONSHIP
}

Sonia Indacochea-Cáceda $a^{1, a, b, c}$

\begin{abstract}
RESUMEN
Introducción: Se entiende por ética a una disciplina filosófica que estudia racionalmente qué es la moral, la virtud, el deber, la felicidad y el buen vivir. En medicina la ética es interna, es inherente a ella, compromete al que ejerce la medicina. En la relación médico paciente, el médico debe estar consciente de que el paciente no tiene dañado solo un órgano, sino que enferma de manera integral. El objetivo de este artículo es revisar los conceptos fundamentales de la Bioética y reflexionar sobre la relación médico paciente en la práctica médica actual en el Perú. Métodos: Revisión de las publicaciones disponibles sobre el tema de estudio: Bioética y relación médico paciente y un análisis enfocado a la realidad de la práctica médica en el Perú, desde la perspectiva hospitalaria docente. Resultados: El concepto de la relación médico paciente ha cambiado en los últimos 25 años más que en los últimos 25 siglos. Existen varios modelos de relación médico paciente: Modelo paternalista, Modelo dominante, Modelo de responsabilidad, Modelo mecanicista, Modelo personalista, Modelo de primacía de la autonomía, entre otros. En el Perú, el impresionante desarrollo de la ciencia y de la tecnología médica, la tendencia a la súper especialización y la transformación de los hospitales en centros de alta tecnología, a menudo teñidos de un sesgo empresarial, han modificado radicalmente la relación médico paciente y la relación entre la medicina y la sociedad. De todos los modelos descritos el de responsabilidad compartida parece el más indicado para desarrollar la relación médico paciente, relación fundamental para el buen resultado del acto médico. Conclusión: Para desarrollar una adecuada relación médico paciente, considerando todos los ángulos y modelos de esta relación es necesario considerar las normas éticas vigentes y vivir algunas virtudes que nos hacen buenas personas $y$, por tanto, buenos médicos. Las virtudes que son importantes en la medicina incluyen la honestidad, integridad, valor, fortaleza, sabiduría, ecuanimidad, justicia, compasión entre otras.
\end{abstract}

Palabras clave: Relación médico paciente; Bioetica. (fuente: DeCS BIREME)

\section{ABSTRACT}

Introduction: Ethics is understood as a philosophical discipline that studies rationally what is moral, virtue, duty, happiness and good living. In medicine ethics is internal, it is inherent to it, it engages the practitioner. In the doctor patient relationship, the doctor must be aware that the patient has not only damaged an organ, but also that he / she is integrally ill. The aim of this article is to review the fundamental concepts of Bioethics and to reflect on the patient physician relationship in current medical practice in Peru. Methods: Review of available publications on the topic of study: Bioethics and patient physician relationship and an analysis focused on the reality of medical practice in Peru, from the hospital teaching perspective. Results: The concept of the doctor patient relationship has changed in the last 25 years more than in the last 25 centuries. There are several models of doctor patient relationship: paternalistic model, dominant model, responsibility model, mechanistic model, personal model, model of autonomy primacy, among others. In Peru, the impressive development of science and medical technology, the trend towards super specialization and the transformation of hospitals into high technology centers, often tinged with business bias, have radically altered the patient-physician relationship and The relationship between medicine and society. Of all the models described, the shared responsibility seems the most suitable for developing the patient physician relationship, fundamental relationship for the good outcome of the medical act. Conclusions: In order to develop an adequate patient doctor relationship, considering all the angles and models of this relationship, it is necessary to consider the ethical standards in force and to live some virtues that make us good people and, therefore, good doctors. Virtues that are important in medicine include honesty, integrity, courage, strength, wisdom, equanimity, justice, compassion among others.

Key words: Patient physician relationship; Bioethics. (source: MeSH NLM)

\footnotetext{
Médico Internista.

Magister en Gerencia y Gobierno en Salud.

Magister en Economía de la Salud y del Medicamento, Hospital Nacional Edgartdo Rebagliati.

Doctoranda en Humanidades (Universidad de Piura).

Correspondencia: Sonia Indacochea Cáceda. Correo: sindacoch@yahoo.es
}

Citar como: Sonia Indacochea-Cáceda. Reflexiones sobre la relación médico paciente. [Artículo de Revisión]. Rev. Fac. Med. Hum. 2017;17(1):84-88. DOI 10.25176/RFMH.v17.n1.753 


\section{INTRODUCCIÓN}

Se entiende por ética a una disciplina filosófica que estudia racionalmente qué es la moral, la virtud, el deber, la felicidad y el buen vivir'.

La ética, al menos la occidental, se fundamenta en dos escuelas: la aristotélica (teleológica) y la kantiana (deontológica). Aristóteles de la antigua Grecia (siglo V antes de C.) sostiene que el fin del hombre es ser feliz y para lograrlo debe alcanzar una vida plena y ésta se logra por una vida virtuosa que es lo que él denomina "vida buena". Es decir, el Hombre por naturaleza tiende a un fin (telos) que es la felicidad. En esta concepción de la naturaleza humana se basa la ética aristotélica. Esta concepción es eminentemente práctica; lo importante para Aristóteles no es "filosofar de la virtud sino que es ser virtuoso". En el siglo XVIII surge Kant que pone en duda esta concepción metafísica del Hombre: que por naturaleza su fin es ser feliz. Para Kant no se puede probar científicamente que la naturaleza del Hombre sea ser feliz y por ende la concepción de vida buena aristotélica. Kant y su escuela deontológica, habla del "imperativo categórico", en él la voluntad es lo fundamental. "Obra de tal manera que tu concepción de lo ético pueda ser probado por leyes universales"

En medicina la ética es interna, es inherente a ella, compromete al que ejerce la medicina, ya que ha hecho un juramento de ayudar médicamente a quien lo necesita y las sociedades científicas y la sociedad en general le exigen su cumplimiento'.

El objetivo de este artículo es revisar los conceptos fundamentales de la bioética y reflexionar sobre la relación médico paciente en la práctica médica actual en el Perú.

\section{MÉTODOS}

Revisión de las publicaciones especificas sobre el tema de estudio: Bioética y relación médico paciente y un análisis enfocado a la realidad de la práctica médica en el Perú, desde la perspectiva hospitalaria docente.

\section{RESULTADOS}

\section{Relación Médico Paciente:}

En la relación médico paciente, el médico debe estar consciente de que el paciente no tiene dañado solo un órgano, sino que enferma de manera integral. Por ello, debemos considerar, que el enfermar conduce a sufrir ${ }^{3}$. El ilustre médico peruano Honorio Delgado considera que los médicos debemos caracterizarnos por un don de humanidad y abnegación, "una suerte de sensibilidad y empatía para el ser de cada hombre enfermo a quien atiende tanto por sí mismo, en su situación concreta, cuanto como prójimo semejante y coparticipe, del destino común colocado en el tiempo frente a la vida, frente a la muerte, frente a lo espiritual e imperecedero" ${ }^{\prime \prime}$. Por tanto, el médico debe tener algunas cualidades como persona, que le permitan entender al paciente como un ser humano que sufre.

\section{Existen algunos datos empíricos ya establecidos:}

A) La percepción de los médicos es que se sienten poco valorados y que la tecnología en medicina atenta en contra de la relación humana médico paciente. Por otro lado los pacientes cada vez son más demandantes, críticos y exigentes. Demandan más consultas y de calidad. Esta relación problemática es por causas externas pero también por la falta de formación de los profesionales de la salud en cómo comunicarse con el paciente.

B) A los pacientes lo que más les frustra es la falta de comunicación con el médico e incluso la priorizan respecto a la receta y exámenes complementarios, que es lo que precisamente el médico le da importancia².

\section{Bioética:}

En el año 1979 aparecen dos escritos claves en el desarrollo de la bioética; el informe Belmont y el libro de Beauchamp y Childress. Ambos se adscriben al modelo principialista de la Bioética.

En estos escritos se plantean los cuatro principios fundamentales de la bioética, a saber, autonomía, no maleficencia, beneficencia y justicia. La autonomía dice relación con la capacidad del paciente para actuar libremente de acuerdo a lo que elige voluntariamente. La no maleficencia se refiere a no hacer daño intencionalmente. La beneficencia es un principio que ha estado presente en todos los códigos éticos de occidente desde la era hipocrática y dice relación con el obrar siempre en beneficio del paciente, aunque fuera sin su consentimiento. Este principio es eminentemente paternalista lo cual ha sido cuestionado al menos como ha sido entendido tradicionalmente. El principio de justicia se refiere a darle a cada cual lo suyo, lo cual implica hacerse cargo en la sociedad de una justicia distributiva lo que equivale al acceso equitativo a los servicios sanitarios. La piedra angular del quehacer médico es la relación médico paciente, que es donde se plasman estos cuatro principios de la bioética. El primero en acuñar el término de bioética es V. R. Potter el año 1971 y la define como "el puente entre las humanidades y las ciencias biológicas". En otras palabras es la disciplina que humaniza y le da sentido a las ciencias biológicas². 


\section{Eras y modelos de la relación médico paciente:}

Los conceptos de la relación médico paciente ha cambiado en los últimos 25 años más que en los últimos 25 siglos. Mark Siegler en 1997 publica los periodos de la relación clínica y los divide en tres periodos:

A) Era del paternalismo o del médico que va del 500 AC a 1960. En este periodo existe una confianza absoluta en el médico, tanto en su capacidad técnica como moral y que además es el único responsable de las decisiones.

B) Era de la autonomía o del paciente, a partir de la década del 60. En 1969 la Asociación Americana de Hospitales, da origen al primer código de derechos de los pacientes. Surge el consentimiento informado: el primer deber de beneficencia del médico es la información. Es recomendable hacer algunas consideraciones sobre este consentimiento:

a) Fuerte impacto de la autonomía la cual está en concordancia con la tradición moral de la modernidad; b) consentimiento informado es ajeno a la tradición médica lo que explica la dificultad de su introducción en el ámbito clínico; c) en vez de entender al consentimiento informado como un proceso democrático y compartido de toma de decisiones, se reduce a la firma de un documento, negando así la validez ética y jurídica del documento; d) el extremo de la autonomía del paciente es equivalente al extremo de paternalismo del médico.

C) Era de la burocracia o del contribuyente. Al médico no sólo se le exige eficacia en tratar a su paciente sino que también eficiencia en gestionar los recursos sanitarios ${ }^{2}$.

Existen varios modelos de relación médico-paciente, pasaremos a mencionar algunos:

- Modelo paternalista: durante siglos, el paradigma hegemónico de esta relación ha sido de carácter paternalista, es decir, una relación vertical y asimétrica, donde el médico ha ejercido un papel activo y autoritario $y$ el paciente uno pasivo $y$ de obediencia. En suma, un vínculo como el que establecen los padres con sus hijos menores y en donde se supone que aquellos siempre quieren lo mejor para éstos, pero sin tener en cuenta su voluntad. En este paradigma paternalista, propio del pensamiento occidental anterior al siglo XIX y que aún prevalece en ciertos lugares, el mandato del médico se ha basado fundamentalmente en dos principios morales, hoy conocidos en la terminología bioética, como beneficencia y no maleficencia ${ }^{5}$. En este modelo prevalece la actitud autoritaria del médico que dirige las acciones, indica y/o realiza los procedimientos diagnósticos terapéuticos, mientras que el enfermo sólo acata las indicaciones, sin que se tomen en cuenta su opinión, dudas o temores. Es una relación tipo sujeto objeto en la cual, aunque se trata de beneficiar al enfermo, no se respetan su autonomía, su libertad, su capacidad y derecho a decidir ${ }^{3}$.

- Modelo dominante: en contraste con el anterior, es el enfermo quien, de acuerdo con sus conocimientos o bien por la información obtenida, pide o exige que, de acuerdo con el diagnóstico establecido, se realicen determinados procedimientos diagnósticoterapéuticos. Es una relación tipo sujeto-objeto que suele ocurrir cuando el médico tratante tiene poca experiencia. En estos casos el abuso de autonomía del enfermo puede ser perjudicial ${ }^{3}$.

- Modelo de responsabilidad compartida: en este modelo se establece una buena comunicación, se informa al enfermo y la familia, lo referente a su enfermedad, el diagnóstico, el tratamiento y el pronóstico, asícomola posibilidad de complicaciones. Se aclaran sus dudas y se trata de disipar sus temores; se discuten las alternativas y en forma conjunta se toma la mejor decisión. En este modelo intervienen la autonomía, la libertad y el juicio del paciente, en relación a lo que quiere o lo que espera, todo ello bajo la orientación del médico tratante 3 .

- Modelo mecanicista En él la atención se lleva a cabo de acuerdo con disposiciones administrativas estrictas; se siguen protocolos de manejo rígidos, el enfermo no elige al médico tratante y no siempre es atendido por el mismo médico lo cual interfiere en la relación médico paciente. El exceso de burocracia y trámites administrativos retarda el tratamiento y deteriora la relación, que es de tipo sujeto objeto. Este modelo es frecuente en la medicina institucional, aunque también ocurre hoy día, en la atención proporcionada por empresas prestadoras de servicios y por compañías de seguros. La terminación de la relación médico paciente puede darse por la falta de colaboración del enfermo o sus familiares, falta de empatía o incompatibilidad en sus valores morales, imposibilidad física o técnica del médico para atender al enfermo, falta de los recursos materiales y humanos para una adecuada atención, interferencia de los familiares, persona responsable u otros médicos, petición explícita del enfermo, familiares o del propio médico ${ }^{3}$. 
- Modelo personalista. Se trata de un modelo en que el eje es el bien del paciente en una relación de confianza. Tanto el médico como el paciente aúnan voluntades en la búsqueda de este bien. La relación se da en el ámbito de la confianza mutua y del debido respeto, que incluye la información y participación del paciente en las decisiones. Este modelo coloca en el centro al paciente y al médico como personas humanas 5 .

- Modelo de primacía de la autonomía. modelo que tiende a absolutizar la autonomía del paciente. Surge, en parte, como reacción a los modelos paternalistas tradicionales ya excesos cometidos por cierta arrogancia de los médicos. En su concepción más extrema, llega a afirmar que el médico debe hacer lo que el paciente cree que es bueno para su salud. El bien del paciente pasa a ser algo subjetivo. No se reconoce un bien objetivo en cuanto a la salud y vida del paciente. El bien de éste, su salud y su vida, en vez de considerarse un bien objetivo, que tanto él como el médico buscan, se transforma en una preferencia subjetiva del enfermo, que el médico debería aceptar y seguir. De esta forma se podría llegar a exigir al facultativo que realizara actos contrarios a la esencia de la medicina ${ }^{5}$.

\section{DISCUSIÓN}

En el Perú, el impresionante desarrollo de la ciencia y de la tecnología médica, la tendencia a la súper especialización y la transformación de los hospitales en centros de alta tecnología, a menudo teñidos de un sesgo empresarial, han modificado radicalmente la relación médico paciente y la relación entre la medicina y la sociedad ${ }^{4}$.

De todos los modelos descritos el de responsabilidad compartida parece el más indicado para desarrollar la relación médico paciente, relación fundamental para el buen resultado del acto médico. Del modelo personalista rescatamos la relación de confianza necesaria para una adecuada relación médico paciente. El elemento fundamental para una buena relación es la confianza, y este primer paso lo iniciaba el paciente al escoger a su médico para confiarle su bien más preciado: su salud. El paciente contaba con que su enfermedad y algunos aspectos de ella permanecerían sólo en el conocimiento de él y de su médico ${ }^{3}$.

Hoy se ha perdido credibilidad entre un segmento de la sociedad, de tal forma que la relación médico paciente se ha llenado de desconfianza mutua que propician una vigilancia de la actuación del médico por parte del paciente, sus familiares o sus abogados ${ }^{3}$. La atención del médico ha de comenzar de manera verbal, informando al paciente sobre lo que se le debe hacer. El médico debe ser consciente de que el primer deber de la beneficencia es la información. Vinculado con ello está el derecho del enfermo a la decisión o consentimiento informado, de ahí que este sea el nuevo rostro de la relación médico paciente ${ }^{6}$. La confianza se desarrolla cuando el paciente recibe la información necesaria por parte del médico. El papel del médico es de un asesor y educador que permite al enfermo tomar decisiones informadas ${ }^{3}$.

Algunas de las tendencias actuales que amenazan la relación médico pacientes son: primacía de la tecnología, con respecto el lado humano de la atención, no disponibilidad de tiempo suficiente para la atención por gran presión asistencial, cambio frecuente de profesionales en las instituciones de salud por la tendencia a la "especialización" disminuyendo las relaciones de largo plazo con los pacientes, aumento de las expectativas de los pacientes en cuanto a información y/o participación ${ }^{5}$.

La entrevista médica para realización de las historias clínicas, las consultas ambulatorias así como las visitas médicas hospitalarias debe ser momentos entrañables de dialogo, afecto y confianza entre el médico (o estudiante) y el paciente. No se entiende estar atentos a otras cosas que no sean el paciente mismo. No es momento para distracciones, ni celulares, el centro de la atención es el paciente mismo.

La preocupación del médico se centraba en el bien del paciente, ahora por concepto de justicia se tiene que optimizar los recursos sanitarios. Adquiere relevancia el principio de justicia distributiva de la ética principialista, ahora el médico tiene un doble rol, a saber, uno individual cual es sanar al paciente y otro colectivo o social cual es; optimizar los recursos sanitarios para lograr una mejor justicia distributiva ${ }^{2}$.

Es necesario, en un país donde las necesidades son grandes y los recursos limitados, el uso correcto con equidad de los recursos disponibles por parte de los médicos. Puig-Junoy considera que la eficiencia corresponde a lograr el máximo nivel de salud a partir de unos recursos dados o cuando al comparar alternativas que generan el mismo beneficio, se opta por la menos costosa, así las actividades de las evaluaciones económicas en salud consisten en realizar la medida de los costos y las consecuencias de las diferentes opciones planteadas para tomar decisiones sobre elegir programas, tecnologías o medicamentos, 
por lo tanto siempre implican comparar en forma juiciosa para así poder optar por la mejor alternativa ${ }^{7}$. Los aspectos económicos también podrían informarse al paciente, por ejemplo, alternativas de tratamiento y costos, para una adecuada decisión por parte del mismo.

\section{CONCLUSIÓN}

Para desarrollar una adecuada relación médico paciente, considerando todos los ángulos y modelos de esta relación es necesario considerar las normas éticas vigentes y vivir algunas virtudes que nos hacen buenas personas $y$, por tanto, buenos médicos. El código internacional de Ética Médica considera los siguientes deberes de los médicos que debemos considerar todos los días desde que empezamos a estudiar esta profesión hasta el final de nuestras vidas: recordar siempre la obligación de respetar la vida humana, considerar lo mejor para el paciente cuando preste atención médica, debe a sus pacientes toda su lealtad y todos los recursos científicos disponibles para ellos y cuando un examen o tratamiento sobrepase su capacidad, el médico debe consultar o derivar a otro médico calificado en la materia, respetar el derecho del paciente a la confidencialidad y prestar atención de urgencia como deber humanitario9. Las virtudes que son importantes en la medicina incluyen honestidad, integridad, valor, fortaleza, sabiduría, ecuanimidad, justicia, compasión entre otras ${ }^{10}$.

\section{Financiamiento: Autofinanciado.}

Conflicto de interés: La autora declara no tener conflictos de interés en la publicación de este artículo.

Recibido: 30 de enero del 2017

Aprobado: 06 de marzo del 2017

\section{REFERENCIAS BIBLIOGRÁFICAS}

1. Drane J. La Historia de la Bioética: Perspectiva Personal. Pag. 21-31. Bioética Clínica. Beca, JP; Astete, C. Editorial Mediterráneo. 2012.

2. Celendon C. Relación Médico Paciente. Rev. Otorrinolaringol. Cir. Cabeza Cuello 2016; 76: 51-54.

3. Arrubarrena Aragón V.M. La relación médico-paciente. Cirujano General 2011:Volumen 33, Supl. 2. S122-S125. www.medigraphic.org.mx

4. Mendoza A. Fundamentación de la enseñanza de la ética médica y la bioética clínica. Acta Méd Peruana. 2009; 26 (2):131-34.

5. De la Uz Herrera ME: La Relación médico-paciente y su panorama actual. Bioetica Enero - Abril 2010: 21-25.

6. Rodríguez Arce M. Relación médico-paciente. La Habana: Editorial de Ciencias Médicas; 2008.
7. Oyuela ME.: La importancia de la reconciliación entre Eficiencia y Equidad, para los sistemas de salud, desde una perspectiva ética. Rev. latinoam.bioet. / ISSN 1657-4702 / Volumen 10 / Número 2 / Edición 19 / Páginas 100-107 / 2010.

8. Delgado H. El médico, la medicina y el alma. Universidad Peruana Cayetano Heredia. Fondo Editorial. Tercera Edición. 1992.

9. Código Internacional de Etica Médica. http://www.wma.net/ es/30publications/10policies/c8/

10. Pellegrino ED. Virtues in medical practice. New York: Oxford University press. 1993

Revisado por: Dr. Jhony De La Cruz (Docente de la Facultad de Medicina de la Universidad Ricardo Palma) y Dra. M.Pilar Bandrés (Docente de la Facultad de Medicina de la Universidad Peruana Cayetano Heredia).

\section{Indizado en: latindex}

http://www.latindex.org/latindex/ficha?folio=14280

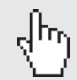

